\title{
ETNOMUSICOLOGÍA, EN TONO FILOSÓFICO: UNA REFLEXIÓN MUSICAL SOBRE EL DEBATE ENTRE EL PENSAMIENTO ANALÍTICO Y EL CONTINENTAL
}

\author{
Jordi Raventós Freixa \\ Universidad de La Rioja
}

\begin{abstract}
RESUMEN: En el presente texto pretendo mostrar cómo la idea de música prevaleciente en la etnomusicología en cuanto disciplina depende de la postura previa que se adopte en el marco del contraste tendido entre las Ilamadas filosofía analítica y la filosofía continental. El debate se traza sobre la cuestión de si la perspectiva dislocada y multifocal de la música característica de la etnomusicología se justificaría por la tensión tendida entre esta polaridad filosófica. Para ilustrar este recorrido teórico se comenta diversos ejemplos en los que dicha problemática se materializa: el método cantometrics, desarrollado por el norteamericano Alan Lomax, como ejemplo de una aproximación analítica de cuño estadístico, y la célebre etnografía musical de Steven Feld Sound and Sentiment, el la que el autor acude a los principios de la filosofía continental para trenzar su projecto. En último extremo, la visualización de las problemáticas que se afrontan desde los puntos de vista convocados nos va a permitir establecer algunas conclusiones abiertas sobre las condiciones disciplinares en las que la etnomusicología aborda la cuestión de qué es música.
\end{abstract}

Palabras clave: Etnomusicología, filosofía de la música, cantometrics, etnografía, filosofía analítica, filosofía continental.

\section{ETHNOMUSICOLOGY, SOUNDING PHILOSOPHICAL: SOME MUSICAL REFLECTION ON THE DEBATE BETWEEN ANALYTIC AND CONTINENTAL THOUGHT}

ABSTRACT: In this text I intend to show how the prevalent idea of music in ethnomusicology as a discipline depends on the previous position is adopted 
under the contrast between the analytic philosophy and continental philosophy. The debate on whether the perspective of music as object dislocated and multifocal characteristic of ethnomusicology be justified by the tension lying between this philosophical polarity. To illustrate this theoretical route I discussed several examples in which this problem is embodied: the cantometrics method, developed by Alan Lomax, as an example of an analytic statistical approximation, and the celebrated musical ethnography Steven Feld Sound and Sentiment, on which the author goes to the principles of continental philosophy to braid your project. Ultimately, the display of this problems that face from the analytic and continental philosophy will allow us open some conclusions about disciplinary conditions in which ethnomusicology addresses the question of what is music.

Keywords: Ethnomusicology, philosophy of music, cantometrics, ethnography, analytic philosophy, continental philosophy.

"Cada ciencia concreta tendería a reproducir, desde su interior, la propia filosofía, es decir el saber reflexivo, vasto y genérico, del cual proviene"

Franca D'Agostini (2000: 542)

La etnomusicología es una disciplina cuyos practicantes, como quien se ocupa en una labor no muy común, nunca se ven dispensados de aclarar los detalles de su actividad. El prefijo etno, proveniente del griego ž $\theta$ vo indica ya su característico trazo orbital en torno al conocimiento de la música según su dimensión etnográfica. No obstante, en un primer nivel, esta idea suscita una definición de la disciplina muy marcada por los objetos típicamente tratados, en este caso, lo que ha llegado a denominarse "músicas del mundo". $\mathrm{Y}$, ¿no es su orientación metodológica lo que substancializa las disciplinas? A pesar de su clara afiliación etnográfica ya anunciada en el nombre de la disciplina, no tenemos aquí más fáciles las cosas. Dice Ramón Pelinski que, gracias su poco desarrollado sentimiento de identidad y de jerarquía disciplinaria, a la etnomusicología "no le importa ponerse trajes ajenos, a condición de que le queden bien" (Pelinski 2000: 297). Estos trajes son los de la antropología y la etnografía, la lingüística y la semiología, la sociología, las ciencias cognitivas, los estudios culturales, y, en general, todas aquellas disciplinas que pueblan los departamentos de ciencias humanas y sociales.

Si este impulso interdisciplinar forma parte del mismo ADN de la etnomusicología, la respuesta fácil a la cuestión de qué es música en esta perspectiva consistiría en parafrasear a Aristóteles y afirmar que la música, como el Ser, se dice de muchas maneras. Sin embargo, en la etnomusicología, esta diversidad ontológica proviene de otorgar a su objeto de estudio el carácter de las "figuras ambiguas" de la psicología gestáltica, en las que el objeto representado cambia 
de configuración a medida que cambia la mirada del observador, vestida con el traje prestado del fondo de armario de las diversas disciplinas.

Pero esta metáfora textil nos ha de recordar también que el patronaje disciplinario recorta la realidad según variados principios. De esta forma, en la etnomusicología hallamos ingresada por la vía de esta interdisciplinariedad una contraposición problemática más general materializada en dos modos de poner en juego la praxis del pensamiento, cuya dualidad se reproduce en el interior de las diversas praxis disciplinarias: un modo científico, fundado sobre la lógica y los resultados de las ciencias naturales y exactas, y un modo que considera determinante la dimensión histórica y situada de todo quehacer humano (D'Agostini 2000: 24). Entendida en su trazo mayor como la antítesis entre la cultura científica y la cultura humanística, esta divergencia ha cristalizado en los ámbitos filosóficos como una contraposición entre las Ilamadas filosofía analítica y filosofía continental. A su vez, transferido a la etnomusicología, este contraste filosófico se manifestaría, también en su trazo más grueso, a través del desarrollo de una etnomusicología de corte más analítico y sistemático, y una etnomusicología de corte más antropológico e interpretativo. En su trazo más fino, dicha contraposición supone para los estudios musicales el abordaje de cuestiones de gran calado estético y epistemológico y, en consecuencia, comporta la reflexión sobre las condiciones de las aportaciones de la etnomusicología a la cuestión de qué es música.

Partiendo de estas cuestiones, en este texto pretendo mostrar cómo la idea de música de la etnomusicología en cuanto disciplina depende de la postura previa que se adopte en el citado marco del contraste tendido entre el pensamiento analítico y el continental. Si la etnomusicología presenta una perspectiva dislocada y multifocal de la música, zésta se justificaría por la tensión tendida entre esta polaridad filosófica?

Para iniciar el debate, expondré en primer el trazo más general de la etnomusicología como disciplina, para seguidamente pasar a comentar las peculiaridades de esta dicotomía filosófica vista desde el prisma de la discursividad etnomusicológica. Concretamente, para ilustrar este recorrido teórico recurriré a diversos ejemplos en los que dicha problemática se materializa: en primer lugar, me referiré al método cantometrics, desarrollado por el norteamericano Alan Lomax (1915-2002), como ejemplo de una aproximación analítica de cuño estadístico diseñada para hallar determinaciones estilísticas a partir de correlaciones entre rasgos musicales y fenómenos sociales. A continuación, como ejemplo de trabajo etnomusicológico trenzado según los principios de la filosofía continental, comentaré un texto de Steven Feld, basado en su célebre etnografía musical de los kaluli de Papúa-Nueva Guinea (1982, 3ª ed. 1990). Por último, la visualización de las problemáticas que se afrontan desde los puntos de vista presentados nos va a permitir establecer algunas conclusiones abiertas sobre las condiciones disciplinares en las que la etnomusicología aborda la cuestión de qué es música. 


\section{La etnomusicología en la academia}

Entonces, ¿qué propone en general la etnomusicología en el seno de las instituciones académicas? La fórmula que funciona como la genuina "denominación de origen" de la disciplina se reproduce en la web de la Society for Ethnomusicology (SEM): "Ethnomusicology is the study of music in its cultural context" (SEM). Estas ideas se repiten casi literalmente en la página de la SIBE. Sociedad de Etnomusicología, cuando ésta se define como "una sociedad científica que reúne a investigadores y estudiantes interesados en la música entendida como hecho cultural y en las relaciones de la música con sus contextos sociales" (SIBE). Estas fórmulas definitorias responden a dos cuestiones principales enraizadas en el desarrollo de la disciplina: la articulación de la misma denominación de "etnomusicología" y su definición como disciplina ligada al método etnográfico y a la antropología.

El término etnomusicología fue utilizado por primera vez por el holandés Jaap Kunst en el subtítulo de su libro Musicologica: A Study of the Nature of Ethnomusicology, Its Problems, Methods, and Representative Personalities (Ámsterdam, 1950, retitulado Ethnomusicology, 1955). La obra se situaba de una forma crítica en un contexto de antagonismo entre la naciente orientación antropológica de la disciplina y la precedente "musicología comparada", el campo de estudios dedicado al estudio comparativo de las obras musicales de los distintos pueblos del mundo. La musicología comparada se irradió desde el Instituto de Psicología de la Universidad de Berlín gracias, sobre todo, al impulso del psicólogo y filósofo Carl Stumpf (1848-1936), quien logró ampliar la institución en 1901 a través de la fundación del Berliner Phonogramm-Archiv, con Erich von Hornbostel (18771935) al frente. La promulgación de las leyes raciales en Alemania en 1933 y el posterior estallido de la Segunda Guerra Mundial supusieron la interrupción de las actividades y, finalmente, el exilio o la emigración a los Estados Unidos de importantes investigadores europeos. Desplazándose el centro de gravedad de los estudios hacia América, la investigación se desarrolló mediante la influencia del antropólogo Franz Boas (1858-1942) quien, a través de su prolongado magisterio en la Columbia University, había logrado promover una aproximación antropológica al hecho musical con un marcado acento particularista.

Cuando a principios de los cincuenta Jaap Kunst publicaba sus estudios ya existía una incipiente línea de estudios musicales orientados por la antropología americana de posguerra. En este ambiente, Kunts propuso el añadido del prefijo etno en substitución del adjetivo alusivo a método comparativo, ya que, mientras que éste era constitutivo de todas las investigaciones científicas, el término alternativo de "etnomusicología", en cuanto ligado al método etnográfico y al fieldwork, lograba representar más claramente la especificidad de la disciplina a través de la idea del contacto con los "nativos". Mientras que esta misma idea presidía la constitución en 1955 de la ya citada Society for Ethnomusicology (Myers 2001: 24), la publicación en 1964 de la obra de Merriam, The Antropo- 
logy of Music, llegó a considerarse el punto simbólico de partida de una cierta hegemonía de la orientación culturalista de la disciplina cuya fórmula definitoria, "estudio de la música en la cultura", hemos visto aparecer en las citadas web a modo de frontispicio declarativo.

Se diría, vistas las formulaciones expuestas por las citadas asociaciones profesionales, que el campo disciplinar y el enfoque etnomusicológicos es producto de una clara y eficiente delimitación basada en la perspectiva antropológica de cuño culturalista cuya definición por antonomasia es la expresada por Merriam. Sin embargo, nada más lejos de la realidad. La fórmula resulta cómoda y proporciona una rápida descripción, pero funciona más bien como marca y frontera disciplinaria que, sin embargo, apenas logra concentrar alrededor del término "cultura" la variedad de posiciones que componen el arco antropológico que la etnomusicología ha llegado a abrazar. A la vez, esta definición minimalista tampoco permite mostrar el carácter complejo de la etnomusicología que Merriam buscaba reflejar cuando proponía una visión de la disciplina basada en la fusión de la antropología cultural, entendida como estudio de los fenómenos de los que la música forma parte, con el estudio de las dimensiones musicológicas de su objeto (teoría y análisis de la música) (1964: 17). Sin embargo, es en esta dimensión de relación compleja entre análisis musical y etnografía, y entre análisis formal de rasgos y análisis contextual de relaciones, que nos hallamos en un punto decisivo en el que, en el campo disciplinario de la etnomusicología, se muestra la cuestión más general del debate de las posiciones epistemológicas y ontológicas trazadas por el juego entre las filosofías analítica y continental.

Esta polarización se refleja en el hecho que, a mediados del siglo XX, la antropología particularista y culturalista derivada de Boas y sus herederos tenía que competir con las ideas evolucionistas de la musicología comparada y con antropologías mucho más formales y de orientación cientifista, como el estructuralismo en Europa, la antropología cognitiva clásica, la neo-evolucionista o la comparativista en Estados Unidos. En este sentido, Franz Boas, desde Columbia, había dirigido su rechazo al método comparativo-estadístico que desde la Universidad de Yale proponía G. P. Murdock (1897-1985), por considerar mecánicas sus operaciones sobre los rasgos culturales, y alejadas de la voluntad de comprensión cualitativa de las culturas "desde dentro". A grandes rasgos, esta misma distancia es la que a mediados del siglo XX se reproducía a través de las diversas posiciones derivadas de la adopción de los modelos lingüísticos que en la arena estnomusicológica competían por señalar la dirección de su contacto con la antropología: por una lado, en tanto que la lingüística parecía haber elaborado un método más seguro, las tendencias que se apoyaban en ella para desarrollar un enfoque más riguroso del diseño de la investigación y la obtención datos; del otro lado, y siguiendo las directrices de Clifford Geertz (19262006), las propuestas que representándose la cultura como un "texto", abordaban una minuciosa reflexión acerca de la forma en que los nativos des- 
cifran, descodifican e interpretan su propia cultura (1987: 57-59). Los partidarios del primer enfoque, enrolados en las filas de la etnociencia, el análisis componencial y la antropología cognitiva, en nombre del rigor científico se declaraban favorables al estudio comparativo de las culturas a través del análisis de correlaciones entre categorías culturales, mientras que los partidarios de la antropología interpretativa de inspiración geertziana perseguía el ideal hermenéutico de la comprensión cualitativa de las culturas, siempre en sus propios términos. La idea de contraposición polarizada en base a algún principio inconmensurable entre ambas se hace patente en el rechazo del mismo Geertz al formalismo matemático de la antropología cognitiva, reconocida ella misma como una especie de espejo de la antropología interpretativa:

Como a primera vista este enfoque parece lo bastante próximo al que estamos desarrollando aquí para que se lo confunda con él, conviene decir explícitamente lo que los divide. [...] La falacia cognitivista — de que la cultura consiste (para citar a otro vocero del movimiento, Stephen Tyler) en "fenómenos mentales que pueden [el autor quiere decir "deberían"] ser analizados mediante métodos formales semejantes a los de la matemática y la lógica" - es tan demoledora para un uso efectivo del concepto de cultura como lo son las falacias del conductismo y del idealismo de las cuales el cognitivismo es una corrección mal pergeñada. (1987: 25-26)

Esta problemática señala su tensión máxima entre los polos de la búsqueda de supuestos esquemas cognitivos universales, por un lado, y, por otro, la atención cuidadosa al nativo en su particular visión del mundo; en este punto creo reconocer en todo su sentido el juego de la filosofía analítica y la continental en tanto que reproducido en el marco de la etnomusicología. Antes de evidenciar estas cuestiones a través de los ejemplos de algunos trabajos de Alan Lomax y de Steven Feld, expongo brevemente los principales aspectos de la polaridad analítica-continental.

\section{Analíticos y continentales}

Aunque enraizado en discusiones anteriores, el debate entre la filosofía analítica y la filosofía continental tomó cuerpo después de la Segunda Guerra Mundial sobre la estela de las herencias filosóficas de Bertrand Russell, George. E. Moore y el primer Wittgenstein, por parte de la filosofía analítica, y de Wilhelm Dilthey, Edmund Husserl y Martin Heidegger, por parte de la filosofía continental (D'Agostini 2000: 36). Bajo sus respectivos rótulos, se encuentran en juego dos modos de concebir los diversos ámbitos del pensamiento, la ciencia y la acción ética. En este sentido, la discusión plantea si la tarea del pensamiento, filosófico y/o disciplinario, trata de un conocimiento fundado sobre la lógica y los desarrollos de las ciencias naturales -perspectiva analítica-, o bien, trata de un conocimiento de orientación humanista, cercano a la literatura y que se sir- 
ve de la historia y el "mundo de la vida" -perspectiva continental-. Se trata de una confrontación conceptual ampliamente presente en los debates contemporáneos, a través de corrientes como el neopositivismo, la fenomenología, la hermenéutica el estructuralismo y el postestructuralismo, el marxismo, la teoría crítica, el posmodernismo y la deconstrucción.

Aunque evidentemente simplificadora, esta contraposición aparece cargada de sugerencias reveladoras, de las cuales las de Richard Rorty quizás sean de las más iluminadoras. La división filosófica que observa Rorty se da entre la concepción analítica de la filosofía como especie ancilar de la ciencia y la concepción continental de la filosofía como crítica cultural: la primera trata de la concepción filosófica que mantienen los pensadores adscritos a esta tendencia, mientras que la segunda apunta a la concepción de una finalidad para los objetos que trata (2007: 124). Otra cuestión esencial que Rorty sugiere de esta pareja de conceptos tiene que ver con su origen diferenciado en las tradiciones kantiana y hegeliana a las que respectivamente se ligan. Gianni Vatimo, en su prefacio al volumen Analíticos y continentales de Franca D'Agostini (2000), ofrece esta caracterización según la perspectiva de Rorty:

La línea kantiana, interesada principalmente en aprovechar las condiciones trascendentales de la posibilidad del conocimiento y en general de la racionalidad, incluida la práctica, se encarna hoy en día en todas las filosofías que concentran su atención sobre la lógica, la epistemología, las formas del saber científico o también del obrar ético con el intento de individuar los rasgos universales y permanentes. Por el contrario, denominamos línea hegeliana, según la propuesta de Rorty, a aquella tendencia que se encuentra en las filosofías que se interesan principalmente por la concreción históricas de las formas de vida, del lenguaje, de los paradigmas científicos, y que, por tanto, colocan en el centro de atención el problema de la historicidad de los saberes y de la propia filosofía, e incluso los éxitos extremos del relativismo. (2000: 14)

Así, para Rorty lo esencial de la dicotomía analítica-continental reside en las consecuencias derivadas de Hegel en su comprensión de la filosofía como enteramente atada a su tiempo:

[...] la diferencia más sobresaliente entre Kant y Hegel es que Hegel no piensa que la filosofía pueda elevarse sobre las prácticas sociales de su tiempo y juzgar su deseabilidad por referencia a algo que no sea en sí mismo una práctica social alternativa (pasada o futura, real o imaginada) [...] para Hegel [...] no hay normas que no sean las de alguna práctica social. (2007: 23)

Uno de los ejes por el que se desarrolla la filosofía continental frente al programa analítico reside, entonces, en la consideración del trabajo del pensamiento como conectado a la historia, a los problemas que se entienden como 
manifestaciones de las inquietudes culturales y éticas de las personas en un momento determinado, y sus cambios como cambios de la sociedad (Bowie 2009: 81). En su ensayo "Analytic and conversational philosophy", perteneciente al volumen Philosophy as cultural politics (2007), Rorty expone su alejamiento de la filosofía analítica precisamente en nombre de la historicidad interpretable de los hechos:

Prefiero la filosofía conversacional a la analítica, así definida, porque prefiero a los filósofos que son lo suficientemente historicistas como para pensarse a sí mismos más como tomando parte de una conversación que como practicando una disciplina casi científica. (2007: 126)

En el ámbito de la etnomusicología, esta problemática se deja traslucir, por ejemplo, cuando Jeff Todd Titon expone un metacomentario acerca del título de un texto suyo, incluído en la obra colectiva The Cultural Study of Music: A Critical Introduction (2003, 2 $2^{\mathrm{a}}$ ed. 2012):

El título de este capítulo Textual Analysis or Thick Description? se me asignó a mí por los editores del libro, y en su formulación sugiere una distinción entre el análisis científico y la interpretación humanista. (Titon 2012: 236)

El juego entre la filosofía analítica y la continental puede definirse, entonces, como propensión hacia la ciencia explicativa, o bien, hacia la interpretación comprensiva: por ello, en él se debate todo el trasfondo filosófico occidental que va a ser vertido en el marco de la etnomusicología. Esta dicotomía, se juega así sobre el hiato entre un trayecto analítico en que la concepción del pensar propende a la comprensión de lo real como un espacio de entidades observables, designables y reconstruible en descripciones y explicaciones sujetas a método, y otro trayecto hermenéutico en el que en la textura de lo real se reconoce una dimensión dinámica del fenómeno, una dimensión del ser-constituído del sentido (Sáez Rueda 2002: 30-31). Lo que hay que aclarar en la visión analítica es el estatuto de hechos positivos del mundo, en nuestro caso, el mundo de la música, mientras que en la visión continental lo que hay que aclarar es el proceso de constitución de mundos en los que la música es a la vez parte y resultado de la construcción social.

Para concretar la visión de estos principios he escogido los trabajos de dos autores muy significados en la historia de la etnomusicología, Alan Lomax y Steven Feld. En primer lugar, comento el proyecto cantometrics de Lomax, el cual muestra el trasfondo analítico de su visión etnomusicológica. Posteriormente, como muestra de una aproximación desde el pensamiento continental, comentaré la etnografía musical de Steven Feld en Papúa Nueva Guinea, muy influenciada por la antropología simbólica. 


\section{El proyecto cantometrics de Alan Lomax}

El proyecto cantometrics de Alan Lomax contituye un estudio comparativo en la forma de la antropología transcultural. En su recensión de uno de los libros de Lomax, Locke cuenta que en 1953, durante uno de sus "field recording" en España, Lomax pudo advertir la diferencia entre los estilos de canto del norte y el sur de la península y sus correspondientes divergencias en destacados patrones culturales (1981: 527). Simples observaciones como estas le pudieron sugerir la idea inicial que Lomax describió en Folk song style and culture (1968, reed. 2000) uno de sus textos principales:

el estilo de canto preferido por una cultura refleja y refuerza aquel tipo de comportamiento acorde con su principal esfuerzo de supervivencia y con sus instituciones centrales de control social. (1968: 133)

En esta línea, en los años 60 Lomax y sus colaboradores plantearon un programa de investigación musical transcultural que fue bautizado como cantometrics, entendiendo con ello un sistema dirigido al registro y evaluación de rasgos musicales de los estilos de canto en el mundo (tipologías de emisión vocal, vinculación entre participantes, ornamentaciones y tempos, y así hasta treinta y siete parámetros observables aisladamente unos de los otros) para buscar sus correlaciones con destacados patrones culturales (Locke 1981: 527).

En la fundamentación de su trabajo, orientado por el cientificismo del análisis, la hipótesis que Lomax hizo valer en su texto clásico de 1962, Song Structure and Social Structure (trad. 2001), se plantea como sigue:

cuando un estilo musical distintivo y consistente vive en una cultura o atraviesa varias, se puede suponer la existencia de un conjunto característico de necesidades o impulsos emocionales que son de algún modo satisfechos o evocados por esta música. Si tal estilo musical se produce con un patrón de variación limitado en un medio cultural similar y a lo largo de un extenso período de tiempo, es posible asumir que ha existido un patrón expresivo y emocional estable en un grupo A en una área $\mathrm{B}$ a través de un tiempo T. (Lomax 2001: 298)

En su parte más práctica, la metodología analítica del cantometrics consistía en la determinación del conjunto de rasgos musicales predeterminados, indexados en escalas de evaluación hasta un número de treinta y siete parámetros presentados mediante un libro de codificación; este libro ocupa cuarenta páginas del tercer capítulo de Folk song style and culture (1968). Evaluada cada variable de acuerdo a los parámetros indexados, los miles de ejemplares musicales de todas las culturas del mundo podían ser catalogados según un perfil resultante para cada caso, para posteriormente buscar su correlación respecto de las variables asimiladas a las categorías culturales tales como el grado de 
estratificación social o el grado cooperación en la actividad económica, entre otras (Lomax 2001: 301 ss.).

Para realizar este programa, Lomax se valió de los desarrollos estadísticos de la antropología comparativa transcultural de George Peter Murdock, a quien ya hemos aludido con motivo de su controversia con Franz Boas. La historia de esta corriente antropológica se remonta a la organización por Murdock, en la Universidad de Yale durante el periodo de entreguerras, de las bases de datos etnográficas conocidas como Cross-Cultural Survey, reorganizadas después de la Segunda Guerra Mundial como Human Relations Area Files (HRAF). La iniciativa se desarrollaba en un ambiente intelectual en que los científicos sociales y psicólogos conductistas se hallaban comprometidos con el desarrollo de una teoría unificada del comportamiento. Estas bases de datos catalogaban etnografías de texto completo o datos etnográficos que, indexados bajo epígrafes uniformes de numerosos culturas de todo el mundo, permitían gestionar gran cantidad de información sobre rasgos culturales en vistas a su comparación transcultural; en este marco, la creación de un cuerpo organizado de datos sobre sociedades y culturas humanas, ampliado posteriormente por Murdock y sus colaboradores con otras muestras siguiendo procedimientos diferentes, como el Ethnographic Atlas (1967) y el Standart Cross-Cultural Sample (1969), era un recurso esencial para la construcción y la evaluación crítica de esta teoría como proyecto con pretensión científica (Goodenough 1994: 307-309). También como medio de pretensión científica, este programa antropológico se valía de una metodología analítica basada en herramientas estadísticas dirigidas al hallazgo de correlaciones entre variables: dando cuerpo a su proyecto cantométrico dentro de estos parámetros, Lomax y sus colaboradores recogieron la convicción que sustentaba esta escuela antropológica, la idea de que la aproximación científica requiere un sistemático estudio transcultural comparativo a partir de los recursos matemáticos y estadísticos que conformaban su método (ib. 307).

En este sentido, el cantometrics es un estudio de correlaciones en tanto que se busca una indicación de la fuerza y la dirección de una relación lineal entre dos variables estadísticas, en este caso un estilo musical y unos rasgos culturales. Un ejemplo fácil de ello es la forma en que Lomax presenta su comparación de los estilos de canto de los pigmeos africanos y de Europa Occidental. En cuanto a los pigmeos, Lomax encuentra un correlato entre su estilo vocal relajado, manifestado a través de una polifonía estructurada con el procedimiento del hoquetus y el recurso del yodel y, según la clásica etnografía sobre los pigmeos de Colin Turnbull (1924-1994) de la cual se valió, su estilo social cooperativo:

Nuestros estudios codificados revelan que un alto grado de integración coral está siempre ligado a un modo relajado y abierto de vocalización [...] La empatía vocal de los pigmeos parece corresponder al estilo cooperativo de su cultura. (2001: 312) 
Por otro lado, la evaluación cantométrica de la canción folklórica europea le permitió afrontar una comparación de ésta con el citado modelo africano, apelando en este caso como correlación sociomusical, la variable musical de la performance dominada por un líder sobre una audiencia pasiva, y la variable sociocultural del sistema jerárquico imperante en las relaciones típicas de esta cultura, visible desde la cadena de montaje fordista hasta en la orquesta sinfónica, todas ellas actividades que dependen "de una serie de órdenes claras y explícitas" (2001: 316). Con estos argumentos, la intención científica de Lomax es dar cuenta de estas correlaciones basándose en la consistencia estadística que establece que a más jerarquía social, menos integración social en el canto, y a la inversa. En su obra principal, Folk song style and culture (1968), Lomax presenta diversos análisis estadísticamente mucho más complejos a partir de los perfiles producidos en las hojas de codificación cantométricas y los datos masivos tomados de los HRAF con los cuales expone las correlaciones resultantes.

En definitiva, la pertinencia de los resultados a los que llega Lomax se halla en un ámbito de realidad que en último extremo es hipotético; en tanto que se busca correlacionar variables, el método estadístico cantométrico pretende establecer la probabilidad, o coeficiente de correlación determinada estadisticamente, de que, dados unos elementos o factores con una puntuación determinada en una variable (un perfil musical), se de una correlación con otros elementos de una segunda variable (un factor o rasgo sociocultural) (vid. Murdock-White 2006). Vale la pena destacar aquí el sentido que Lomax se encarga de remarcar a propósito de su método en tanto que propensión hacia un estilo de pensamiento preciso y científico, fundamentado en el rigor estadístico que encaja con la ambición analítica:

De este modo podríamos esperar una musicología científica que pudiera hablar con cierta precisión acerca de actitudes emocionales formativas que penetran culturas y operan a través de la historia. (2001: 298)

A pesar de su esfuerzo fundamentador, la musicología comparativa pareció agotarse durante el último tercio del siglo XX con el proyecto de Alan Lomax (Titon 2012: 240). En su misma época, paralelamente a la antropología comparativista y transcultural, otras tendencias enraizadas en el antiguo particularismo boasiano pugnaban por su reconocimiento: en este marco, a la síntesis comparativa se le oponía el análisis de lo peculiar, o a la búsqueda tipológica de los rasgos comparables, el ideal de la expresión de la cultura en sí misma. Estas oposiciones conceptuales Ilegaron a cristalizar en las formas antropológicas que proliferaron a mediados del siglo XX. Sin embargo, ninguna logró una mayor resonancia que la oposición entre las aproximaciones "emic" y "etic" (vid. Darnell 2008: 47). Este par conceptual exponía la polarización entre dos formas posibles de ver las distinciones fenoménicas, según si son vertidas a partir del punto de vista del investigador o del insider o nativo. Desde un contexto de trabajo etnomusicológico, Gerard Kubik expone la contraposición de esta forma: 
Investigar desde un punto de vista emic significa analizar un sistema de acuerdo con sus propios componentes significativos. Investigar desde un punto de vista ético, por otro lado, significa analizar uno o más sistemas de forma comparativa en referencia a un marco analítico de conceptos creados por el investigador y proyectado sobre esos sistemas. (1996: 5)

Desde esta perspectiva, los resultados de Lomax podrían ser considerados de carácter etic. Su orientación cientifista, teniendo en cuenta su comparativismo de fuerte componente estadístico, se alineaba con las tendencias que Clifford Geertz tuvo en mente cuando las contrapuso a su proyecto hermenéutico en la famosa frase: "el análisis de la cultura ha de ser por lo tanto, no una ciencia experimental en busca de leyes, sino una ciencia interpretativa en busca de significaciones" (Geertz 1987: 20). La dicotomía percibida entre los "científicos" y los "humanistas" en antropología (Darnell 2008: 47), se reproducía en la arena etnomusicológica, durante la segunda mitad del siglo XX, de una forma paralela al debate emti-emic. En este escenario, Lomax representaba un desarrollo vinculado al pensamiento analítico, de carácter "etic", por su recurso a los métodos neopositistas, su planteamiento del pensamiento y la filosofía como especie ancilar de la ciencia, y, last but not least, por su omisión de los postulados continentales que, según la ya comentada caracterización de Rorty, eran favorables a la consideración de la historicidad interpretable de los hechos. Todo ello situaba al método cantométrico en las coordenadas contrarias a la perspectiva "emic" que sería dominante en la antropología y en la etnomusicología. Esta problemática traza el puente que nos Ileva del trabajo analítico y "etic" de Lomax al siguiente ejemplo etnográfico y "emic" de Steven Feld.

\section{La etnografía en Steven Feld}

Como hemos visto, la visión continental en la etnomusicología se correspondería al esfuerzo de aclarar el proceso de "construcción de mundos" en los que la música es a la vez parte y resultado de dicha construcción. El trabajo de Feld es un ejemplo de cómo este objetivo vendría dado en gran parte de la escritura etnográfica del último tercio del siglo XX como una disminución de la atención hacia la actividad social y una mayor orientación hacia "las categorías, las metáforas y las retóricas incorporadas a las descripciones que los informantes hacen a los etnógrafos de sus culturas" (Marcus-Fischer 2000: 83). En esta línea, la obra principal de Steven Feld, Sound and Sentiment, constituye una etnografía musical cuyo objetivo es la demostración de la siguiente tesis:

las modalidades expresivas de los Kaluli en los lamentos la poesía y la canción en su estructura musical y textual, son representaciones en espejo del círculo simbólico construido por un mito, el de "el muchacho que se convirtió en un pájaro muni. (1990: 14) 
El pueblo Kaluli es uno de los de los cuatro subgrupos de lengua Bosavi que habitan en la selva tropical de las highlands del sur de Papúa Nueva Guinea (1990: 3). Dedicados a la horticultura, Feld describe como su vida musical se relacionaba íntimamente con una vida ceremonial muy prolífica, vehiculada primariamente por las formas vocales: en este contexto, los tambores con parche de piel se hicieron un lugar, sobre todo, como preludio vespertino de una ceremonia nocturna (1990: 334).

La referencia al mito del muchacho convertido en pájaro busca establecer la música dentro de su mundo simbólico. En este sentido Sound and Sentiment es definido por su autor como un estudio etnográfico del "sonido como un sistema cultural", o como "un sistema de símbolos" enraizado en la cultura Kaluli, y se muestra cómo un análisis de modos y códigos de comunicación sonora que conduce a una comprensión de la vida en esa sociedad (Feld 1990: 3). Lo que Feld tiene en cuenta a través del mito de referencia, y en una manera claramente apoyada en el estructuralismo de Lévi-Strauss, es la forma y el funcionamiento de aspectos como el Ilanto, la poética y el canto en su relación con el mundo natural y espiritual de los Kaluli, lo que permite revelar sus expresiones sonoras como encarnaciones de profundos sentimientos (ib.). Todas estas articulaciones aparecen también en otra de las dimensiones musicales Kaluli, la música instrumental del tambor ceremonial, cuyo estudio es presentado por Feld en otro conocido trabajo titulado Sound as a Symbolic System: The Kaluli Drum (1991, trad. 2001). El carácter sintético de este estudio nos permite utilizarlo a continuación para ejemplificar de uan forma más detallada la aproximación etnográfica del autor al fenómeno musical.

Ya que en la cultura Kaluli los nativos expresan el sentimiento a través del sonido que siempre es mediado por las aves, el toque del tambor es asimilado a la intensas pulsaciones emocionales del canto del tibodai (Pitohui cristatus), el cual, a su vez, en ciertas condiciones performativas, evoca la voz de un ñino pequeño muerto llamando al padre (2001: 347). El objeto que Feld recorta para el estudio del fenómeno es toda la construcción simbólica que otorga sentido a la actividad: la mediación mágica que preside el proceso de construcción del tambor, desde la obtención de la madera de magnolia, hasta su vaciado y posterior relleno con elementos especiales y su puesta a punto en consonancia con las prestaciones que, vinculadas al universo simbólico Kaluli, se esperan de él. En el inicio del proceso hay una partida de caza para capturar un pájaro tibodai, cuyas plumas van a ir a parar al interior del tambor. De forma más dramática, la garganta y la lengua del pájaro se colocarán en un puente que separa las dos zonas huecas del interior del tambor, mientras el constructor recita una invocación por la que consigue que la boca y la voz del tambor sean como las del tibodai. Después, mientras se trabaja la forma del tambor, se caza un yobo, un gran lagarto de cabeza angulada (Goniocephalus sp.) para utilizar su piel como parche del membranófono. A continuación se echa sobre este parche algunos pegotes de cera de abeja a los que los Kaluli se refieren metafórica- 
mente como "mierda de la oreja de un perro del bosque". Una vez fijados estos pegotes, reciben otro conjuro por el que se los asimilará a la cualidad de "corazón de un perro del bosque", adquiriendo poder para latir y retumbar como un perro durante la caza. Las pruebas de sonido que vienen a continuación se destinan a la comprobación de la capacidad del tambor para "endurecerse", es decir, alcanzar la cualidad sonora y estética necesaria para ser un tambor válido en su contexto sonoro y mitológico (2001: 338-340).

En la situación performativa, los tambores se tocan a última hora de la tarde como preludio a una gran velada ceremonial. Una vez "endurecido" el sonido, lo que significa que ha comenzado a sonar con fuerza, dos grupos de danzantes evolucionan frente a frente; si el sonido se "ablanda", el pegote de cera es de nuevo ajustado. El "endurecimiento" no es simplemente un requisito de una estética abstracta; es el momento en que la voz del tambor deja de oírse como la voz de un pájaro tibodai y, desde "dentro", desde su reflejo interior, suena como un niño muerto llamando al padre (ib. 347). Los Kaluli asocian a los pájaros todos los sonidos de su entorno, pero estos sonidos tienen un dominio visible y otro reflejo, por lo que son también reflejos de los difuntos: los tambores son voces de tibodai e, internamente, la voz de un espíritu reflejo, un niño muerto (ib. 349) La performance, como las canciones que sonaran por la noche, busca mover los sentimientos de la audiencia arrastrándola a un estado de ánimo nostálgico, sentimental y reflexivo, en el que la experiencia, firmemente anclada en su universo de creencias, pueda sobrepasarlos y provoque intensas lágrimas, e incluso, en la profunda conmoción, la quema del doloroso tambor (ib. 346-347).

Vemos en este caso cómo el trabajo de Feld oscila desde la atención a un punto central, el toque ceremonial del tambor Kaluli, hacia aspectos que van desde la ornitología y la clasificación de las aves, la exploración de las metáforas vertidas en toda explicación Kaluli, el sentido de las ceremonias, las relaciones con los pájaros... Teniendo en cuenta el papel referencial del factor central por cuyo alrededor orbita todo elemento etnográfico, el trabajo de Feld se asemeja en su concepción al de Anthony Seeger en Why Suyá Sing: A Musical Anthropology of an Amazonian People (1987, reed. 2004), en el que el autor buscaba trazar el retrato de la cultura musical a partir del estudio minucioso de una sola ceremonia que es tomada también en su papel referencial. Ambos casos muestran como la antropología de la música se erigía como enfoque prometedor dentro de la heurística etnomusicológica; ambos, partiendo de un mito o una ceremonia, ejemplificaban una aproximación microcósmica que actuaba como si el comparativismo de los grandes registros de áreas culturales se hubiese diluido en favor de una mirada minuciosa e intensiva que enfatizaba el particularismo de cada cultura a partir de pocos datos, lejos de su acopio masivo, pero explotados al máximo en nombre de la interpretación. Si el motivo del etnomusicólogo es hurgar en la significación, entonces, proponer una significación va a depender de la acción interpretativa, es decir, "poner en relación el conocimiento y la epistemología culturales con la experiencia concreta del sonido" 
(2001: 353). Por estas razones, el trabajo de Feld es un ejemplo de lo que Marcus y Fischer denominaron "etnografía realista", en la que los marcos analíticos iniciales tendían a tomarse del "mundo público del sentido común", o en términos filosóficos, dentro del marco fenomenológico del "mundo de la vida": los textos realistas, ligando la etnografía al trabajo de campo y a la observación participante, constituyeron una de las herencias de la tradición antropológica mayor cuyo inicio se le atribuye a Bronisław Malinowski (1884-1942) (2000: 93).

Frente al tipo de validez que en la musicología comparativa y el cantométrics provenían de su metodología cualitativa, la validez de los textos etnográficos realistas residía en el hecho que sus autores habían estado "presentes", atrapando empáticamente el sentido de acontecimientos y gestos, dando un paso atrás para situarlos en sus contextos más amplios, de forma que "la experiencia del investigador puede servir como la fuente de la autoridad en el trabajo de campo" (Marcus-Fischer 2000: 94; Clifford 1995: 54). En su monografía, Feld describe en este sentido vivencial su experiencia de la música en común con los nativos, intentando aprender su forma de componer, al tiempo que busca recursos retóricos para narrar su historia compartida:

Al enseñarme a bailar y tocar el tambor, los Kaluli siempre insistían en que debía sentir el pulso en el pecho y la parte superior de los brazos, no solamente en la parte baja de la mano y los dedos. (1990: 343)

La filiación en la filosofía continental de todo este trabajo debe ya de ser clara, como también lo es desde la confesión de las influencias que Feld reconoce en la introducción de Sound and Sentiment:

Los medios analíticos necesarios para esta tarea son diversos, sin embargo, las posiciones intelectuales que he hallado más útiles son el estructuralismo de Claude Lévi-Strauss (1966), la descripción densa de Clifford Geertz (1973) y el paradigma de la etnografía de la comunicación propuesto por Dell Hymes (1974). (1990: 15)

La influencia confesa de Geertz es compartida en numerosos trabajos, y en referencia a ella, Jeff Titton opina que durante los últimos treinta y cinco años "ningún erudito tuvo más impacto en las representaciones etnomusicológicas de personas que hacen música" (Titon 2012: 240). Al mismo tiempo, la referencia a Geertz traza en gran parte el modo de propensión etnomusicológica hacia el pensamiento continental: en la medida que la metodología geertziana de la "descripción densa" consiste en desentrañar estructuras de significación en "textos" culturales y en determinar su campo social y alcance, el estilo analítico de las ciencias sociales cede el terreno a las prácticas interpretativas de las humanidades (Geertz 1987: 24). Es significativo como Tihothy Rice, después de invocar a la hermenéutica filosófica como esencial para su reinvención como etnomusicológo, integra la antropología interpretativa de Geertz en el panteón de tradición continental de la hermenéutica que viene trazada partiendo de 
Wilhelm Dilthey, pasando por Martin Heidegger, Hans-Georg-Gadamer y Paul Ricoeur, e ingresando en la antropología de la mano de Geertz (Rice 1997: 114) Esta invocación a la hermenéutica es coherente con el realismo etnográfico que comparte con Feld cuando, por ejemplo, éste alza su etnografía de la música búlgara sobre la experiencia musical compartida con Kostadin, su maestro de gaida, una gaita búlgara; la comunicación establecida es entendida por Rice no como a través de la superación de "fronteras" culturales, sino a partir de la metáfora hermenéutica gadameriana de "fusión de horizontes", como experiencia de transformación mutua (1994: 85; 1997: 115).

No hay lugar, pues, como vemos en los anteriores ejemplos, para la afirmación en la orientación "continental" etnomusicológica de un sujeto constante que se sitúe por encima del mundo, como "reinando sobre la objetividad" (Rice 1997: 119). En los términos hermenéuticos heredados de Heidegger, ser-en-elmundo es la condición ontológica del yo antes de conocer e interpretar, y lo que el sujeto trata de comprender y manipular son las formas simbólicas construidas como el idioma, el comportamiento social, la vestimenta o la música (ib.). Por ello, dice Feld, el significado musical se coloca esencialmente no "en las notas", sino "en el mundo", y, en este sentido, la escucha de esas notas es algo que "deriva siempre de una imposición social anterior" (2001: 333-353). En el caso estudiado por Feld, "el tropo único y fundamental para toda la acción estética Kaluli es la mediación de los pájaros", por lo que todo el significado de la acción musical Kaluli va a tener que ver con el hecho de poner en práctica sus estrategias estéticas más básicas y sentir de esta forma que el sonido del tambor lleva al corazón mismo de "cómo ser Kaluli" (2001: 350-352).

\section{Comparando posiciones}

Comparando el trabajo cantométrico de Lomax con el etnográfico de Steven Feld, vemos como ambos se desarrollan entre los extremos de una eficiente cientificidad y una evocadora orientación humanística. Siguiendo nuestro argumento, esto refleja su posicionamiento antagónico dentro de la dicotomía analítica/continental. ¿En qué sentido las respectivas posiciones sobre el ser de la música va a depender de este otro posicionamiento? ¿Qué diferencias se trazan sobre el fondo de sus respectivas adscripciones? ¿Qué aspectos de estas cuestiones pueden generalizarse a partir de los ejemplos mostrados?

En primer lugar, la respectiva posición hacia el objeto musical va a depender del grado en que el modelo para toda elaboración del conocimiento sea el de la adquisición. Vemos cómo en la elaboración de Lomax, los datos se "encuentran" en tanto que ya "están ahí", inteligibles siempre que se toque la tecla metodológica oportuna, como una sustancia autosuficiente con propiedades inherentes. En este punto, la idea del conocimiento como adquisición no es coherente con el modelo humanista-continental en el sentido que no contempla el conocimiento como un desarrollo sujeto a su construcción social. En el caso de Feld, 
sobre el fondo de la humanidad compartida de los participantes, los datos se construyen en la interacción en contextos de acción musical que conforman un todo conexo. Por su parte, Lomax parece partir en busca de una objetividad musical que siempre podrá ser adquirido como conocimiento de la realidad; Feld, por otro lado, muestra una actitud de partida dirigida a la comprensión de la subjetividad de toda experiencia cuyo sentido es producto de una construcción de los sujetos. A raíz de esta concepción diferenciada del objetivismo y el subjetivismo, el proceso analítico parte de una postura referencialista y antirreflexiva, que, como vemos en Lomax, elabora a través de correlaciones impersonales, mientras que la postura de Feld favorece la elaboración a través de una atención autoreflexiva de cuño hermenéutico que integra al sujeto del conocimiento en la misma operación cognitiva. Este último aspecto, como elemento del credo continental, entronca con la tesis diltheyana de la implicación del sujeto en los propios datos de la experiencia, y que atraviesa los planteamientos tanto de Husserl como de Heidegger buscando el examen reflexivo de la "fundamentación del yo" en la experiencia del "mundo de la vida".

Para llevar a cabo sus respectivos programas, las habilidades puestas en juego por cada autor se despenden de estas consideraciones iniciales. Para los analíticos, lo esencial es desarrollar habilidades argumentativas y saber buscar y encontrar los datos pertinentes, elaborándolos de forma adecuada a partir de los recursos técnicos disponibles. Para los continentales, la búsqueda es equivalente a la lectura de las acciones, entendiendo la acción como texto, compaginando una narración general en la que cada sector de la cultura es articulado con los otros. En los casos de Lomax y Feld, el método estadístico y la observación participante dan cuenta del desarrollo necesario de estas habilidades diferenciadas. Lomax trata de dar cuenta de un hecho expresado como proposición, cuyo carácter abstracto se da en tanto que su veracidad proviene de sus condiciones estadísticamente estables: su modelo estadístico no busca explicar algo, sino dar cuenta de lo que se establece en la proposición como saber válido. Para Feld, en cambio, el hecho se expresa en su concreción de experiencia vivida, y en la forma narrativa con la que tal expresión puede comunicarse. Para Lomax, los elementos de su elaboración son datos a partir de los cuales mostrar la composición de la realidad, mientras que los elementos para Feld son informaciones dentro de un contexto comunicativo que cabe interpretar para llegar a su comprensión. En este sentido, Feld toma a su cargo la consideración propia de los continentales acerca del proceso literario (la utilización de la metáfora, la figuración, lo narrativo, que en el posmodernismo va a tomar una centralidad temática relevante), cuando expone su trabajo aceptando que la noción de literatura es un aspecto que, afectando a todas las vías del fenómeno cultural, desde las primeras notas, desde las primeras observaciones, hasta el libro ya concluido, permea cualquier trabajo que verse sobre las representaciones culturales (Clifford-Marcus 1991: 29). 
Desde el punto de vista de la teoría del conocimiento, estas cuestiones ilustran los principios contrapuestos de raíz kantiana o hegeliana que Rorty establecía como base de la dicotomía analítico-continental: en una visión kantiana, la experiencia, entendida sobre una base empirista, es un proceso por el que ciertas intuiciones desconectadas van siendo integradas y organizadas, mientras que en una visión hegeliana se afirma una indistinción originaria, un todo, que va siendo atenuada a través de sus distinciones dialécticas (D'Agostini 2000: 29). En este punto, en la visión continental, en nombre de su propensión dialéctica, se va a retraer el carácter dogmático de la propuesta analítica: por ejemplo, la lógica estadística de Lomax se muestra dogmática por tal como, en lo afirmado en sus proposiciones, la estadística pone a prueba su verdad decidiendo por el sí o por no, como un resultado fijo, mientras que para el pensamiento dialéctico la naturaleza de la verdad no es la misma en todas las esferas del saber, en el sentido que la complejidad dialéctica trata el ser como un todo recorrido por diferenciaciones. Por todo ello, en la dicotomía analítica-continental se da una contraposición entre una actitud teórica y otra histórica: la primera, de índole sincrónica, es una actitud calculadora, instrumental y clasificatoria, mientras que la segunda, de índole diacrónica, conlleva la crítica a esta racionalidad por cuanto enfatiza, según la idea de Max Weber, una operatividad técnica de medios dirigida a fines que es distinta al mundo de los sentimientos y valores (1964: 21). En este sentido, a través de la dicotomía analítica-continental se da una alternativa entre un modo lógico-matemático-científico y un modo histórico-literario-humanista.

El primer modo lógico-matemático-científico mantiene un estilo teórico que busca la concreción en el lenguaje formalizado y generalizable para exponer sus propuestas; un ejemplo de ello es la formulación de la hoja cantométrica de codificación. En el modo histórico-literario-humanista se busca un estilo textual que hace uso de argumentaciones no generalizables en el mismo sentido universalizante que en el modo analítico, sobre aspectos locales de grandes unidades de sentido (un mito, una creencia, un instrumento).

En definitiva, en la contraposición analítica-continental se da una disparidad esencial acerca de los límites de lo irracional, en el sentido de dónde se impone la pureza o impureza de la lógica (Agostini 2000: 25). El lugar de la racionalidad en mayúsculas en el estudio de la música es el análisis, de manera que, en su consideración, puede ocurrir que éste se considere una especie de sensatez racional que busca de cada elemento un sentido bien definido, que brinda su regla de economía lingüística para el privilegiado ejercicio de la lógica científica. O bien, a la inversa, también puede ocurrir que otra lógica que no se enraíce en la ciencia quiera delimitar en ésta la inutilidad de sus palabras formalizadas y alejadas de la vida del análisis, proponiendo a cada actividad que se muestre a si misma en la vibración genuina de su experiencia, depurando la conceptualidad de todas las intrusiones de la ciencia. En este punto es adecuado recordar cómo Carl Dahlhaus (1928-1989) ya alertaba de la estrecha relación que se da entre teoría y métodos de análisis por tal como la primera puede ser premisa, finalidad o resul- 
tado del análisis musical (cit. en Pelinski 2000: 128). Esta cuestión se reproduce también en la polarización analítica-continental por cuanto afecta a la relación entre análisis y contexto; el análisis musical puede enfrentarse, absorber o ser absorbido por la idea de contexto según ésta sea conceptualizada como premisa, finalidad o resultado de la investigación (cfr. Samson 1999: 50-51).

En definitiva, a través de la controversia entre el pensamiento analítico y el pensamiento continental, observamos como la cuestión de lo que es música se articula de una forma que es irreductible a su propia polarización: o bien la música es un hecho de estructura, que resitúa todo su entorno como algo extramusical, o bien, es un hecho de cultura, por el que la música se constituye en el marco holístico de relaciones sociales. Sin embargo, dada esta bipolaridad, y a consecuencia de ella, la fundamentación de la etnomusicología puede abrirse a la proliferación de posibilidades y la constante búsqueda de trajes disciplinarios a medida. No quisiera concluir con la imagen de una dualidad irreductible ya que, entre otras razones, no resultaría acorde con su desarrollo: la divergencia, bastante clara entre los años 30 hasta los 60, se ha ido haciendo cada vez más compleja, enriqueciéndose con articulaciones que a veces han delineado incluso escenarios de convergencia (D'Agostini 2000: 24). La metáfora de la inconmensurabilidad hay que matizarla con el trazo de puentes transitables.

En la actualidad, las corrientes musicológicas radicales de raíz continental de las últimas décadas, son objeto de crítica por cuanto han supuesto el abandono de cualquier totalización narrativa en favor de un énfasis en el localismo cultural y una política de la diferencia; según Richard Middleton, el modelo continental se halla en crisis como consecuencia de la transformación de la celebración multicultural de la globalidad en una sensación de impotencia política ante lo que se presenta como una totalidad, el marco actual universal del capitalismo neo-liberal (2012: 70). En este espacio, como reacción al localismo, los viejos temas de la musicología comparada vuelven a estimular el pensamiento de una nueva generación de científicos que abordan el estudio de la expresión musical como conducta gobernada por reglas, una perspectiva que todavía anima enfoques científicos a la música. En una web titulada precisamente Comparative Musicology, activa desde 2012 y coordinada entre otros por Steven Brown, destacado neurocientífico dedicado al desarrollo de una comprensión holística de las bases neuronales, cognitivas y evolutivas de las artes, aboga por el restablecimiento del programa -"The time has come to reestablish the field of comparative musicology"-, en base a estos principios:

En efecto, la musicología comparada discute una serie de cuestiones importantes después de un largo tiempo ignoradas por muchos etnomusicólogos, como la clasificación musical, la cartografía de estilos musicales, la evolución cultural de la música, su universales, su papel como la musicología marcador migratorio, la distribución general de estilos musicales, así como la evolución biológica de la música [...] clasificar las músicas del 
mundo en grupos estilísticos, describir la distribución geográfica de estos estilos, a iluminar las tendencias universales de la música a través de las culturas, y para comprender las causas y los mecanismos que subyacen a la evolución biológica y cultural de la música.

Habiéndose liberado de la estigmatización racista del evolucionismo cultural, Titon expone cómo, con la intención de llegar a nuevas generalizaciones acerca de la música y la cultura que darían respuestas a las grandes cuestiones ("cómo se originó la música, creció y se extendió entre los pueblos del mun$\mathrm{do}^{\prime}$ ), actualmente se "especula una vez más sobre la música como una herramienta adaptativa en la evolución humana (véase Wallin, Merker y Brown 2000)", mientras que "los neurocientíficos cognitivos y etnomusicólogos están haciendo preguntas interesantes acerca de la música, el cuerpo y el cerebro (véase Hallam, Cross y Thaut 2009, y la sección de 'Call and Response' de Ethnomusicology 53 (3) [2009])" (Titon 2012: 240). Es significativo como en esta última publicación citada por Titon, coordinada por su antiguo colaborador Victor Grauer, la ilustración de la portada es una reproducción de una de las viejas hojas de codificación cantométrica.

Dada la polaridad analítica-continental que hemos tratado en este texto en su plasmación en la etnomusicología, no se trata ya de ofrecer como conclusión unas series de razones que decidan por un si o por uno en relación a cada opción. Por el contrario, quisiera trazar una imagen de esta problemática en la que se debate qué es música a través de una figura plástica, la anamorfosis. Según el Diccionario de la Real Academia Española, una anamorfosis o anamorfismo, es una "pintura o dibujo que ofrece a la vista una imagen deforme y confusa, o regular y acabada, según desde donde se la mire". El ejemplo más conocido se halla en el cuadro de Los Embajadores, Ilamado en realidad Jean de Dinteville y Georges de Selve, de Hans Holbein el Joven (National Gallery, Londres, 1533), en el que aparece en primer plano una figura extraña, irreconocible, que en realidad es una calavera muy deformada por una anamorfosis que sólo es reconocible desde una posición determinada de la mirada del observador. El anamorfismo ha sido utilizado por el filósofo esloveno Slavoj Žižek, figura destacada del pensamiento actual, para destacar cómo se trata de un efecto perspectivo utilizado para forzar al observador a un determinado punto de vista preestablecido o privilegiado (1999: 223).

La imagen del anamorfismo proporciona así, una metáfora de lo que la música nos sugiere como objeto y como actividad según las operaciones etnomusicológicas marcadas por su propensión analítica o continental: desde cada posición, la visión musical contraria adquiere "una imagen deforme y confusa, o regular y acabada, según desde donde se la mire", En este sentido, como diría Žižek, el objeto anamorfizado tiene en sí mismo las claves del desciframiento, sólo nos hace falta saber que nuestro propio régimen de mirada tiene un punto ciego, amenazante, cuyo truco es tomarlo en su angulo adecuado. Y no se trata 
de que una misma realidad, como las citadas figuras ambiguas gestálticas, sólo pueda contemplarse desde una perspectiva parcial, sino que nuestro cambio de posición modifica el objeto, la mirada del observador puede modificar la consideración misma del objeto según la posición escogida. Transitando la etnomusicología en su cadencia filosófica, por ejemplo, por el camino de encuentro y desencuentro analítico-continental, se justifica la necesidad del "filosofarse" de las ciencias y las disciplinas, como tomarse un "margen filosófico" en la consideración de aquello que se está tratando (D’Agostini 2000: 542). La etnomusicología, entonces, preservando a toda costa su ADN interdisciplinar, estaría en condiciones de ofrecer numerosos argumentos a un debate que, tomando en serio y como posibilidad el borrón-anamorfismo con que se presenta a la mirada desde el argumento contrario, permita transitar por todos los registros posibles del pensamiento y la emoción, ahora tomando la música como cosa, después como mediación humana.

\section{Bibliografía}

BAKAN, M. B. (2009). "Measuring Happiness in the Twenty-First Century: Ethnomusicology, Evidence-Based Research, and the New Science of Autism". Ethnomusicology 53 (3), pp. 510-518.

BECKER, J. (2009). "Ethnomusicology and Empiricism in the Twenty-First Century". Ethnomusicology 53 (3), pp. 478-501.

BOWIE, A. (2009). "Music aesthetics and critical theory", en Harper-Scott, J. P. E. y Samson, J. (eds.), An Introduction to Music Studies. Cambridge, Cambridge University Press, pp. 79-94.

CLIFFORD, J. (1995). Dilemas de la cultura. Barcelona, Gedisa.

CLIFFORD, J. y MARCUS, G. E. (eds.) (1991). Retóricas de la antropología. Barcelona, Júcar.

Comparative Musicology: Home. Recuperado en http://www.compmus.org/.

D'AGOSTINI, F. (2000). Analíticos y continentales. Guía de la filosofía de los últimos treinta años. Madrid, Cátedra.

DARNELL, R. (2008). "North American Traditions in Anthropology: The Historiographic Baseline", en Kuklick, H. (ed.), New History of Anthropology. Malden, Blackwell, 35-51.

FELD, S. (2001). "El sonido como sistema simbólico: El tambor Kaluli", en Cruces, F., Las culturas musicales: lecturas de etnomusicología. Madrid, Trotta, pp. 331-355.

-, (1990). Sound and Sentiment: Birds, Weeping, Poetics, and Song in Kaluli Expression. Philadelphia, University of Pennsylvania Press.

GEERTZ, C. (1987). La interpretación de las culturas. Barcelona, Gedisa. 
GOOdENOUGH, W. H. (1994). "George Peter Murdock. National Research Council: Biographical Memoirs v. 64". Washington DC, The National Academies Press, pp. 304-319.

HALLAM, S., CROSS, I., y THAUT, M. (eds.) (2009). The Oxford handbook of music psychology. New York, Oxford University Press.

KUBIK, G. (1996). "Emics and Etics: Theoretical Considerations". African Music 7 (3), pp. 3-10.

KUNST, J. (1955). Ethnomusicology. La Haya, Martinus Nijhoff.

LOCKE, D. (1981). "Cantometrics: An Approach to the Anthropology of Music, by Alan Lomax", Ethnomusicology 25 (3), pp. 527-529.

LOMAX, A. (1968). Folk song style and culture. Washington DC, American Association for the Advancement of Science.

-, (2001). "Estructura de la canción y estructura social", en Cruces, F., Las culturas musicales: lecturas de etnomusicología. Madrid, Trotta, pp. 297-329.

MARCUS, G. E. y FISCHER, M. M. J. (2000). La antropología como crítica cultural: Un momento experimental en las ciencias humanas. Buenos Aires, Amorrortu.

MERRIAM, A. (1964). The Antropology of Music. Evanston, Northwestern University Press.

MIDDLETON, R. (2012). "Introduction. Music Studies and the Idea of Culture", en Clayton, M., Herbert, T. y Middleton, R., The Cultural Study of Music: A Critical Introduction. New York, Routledge, pp. 37-78.

MURDOCK, G. P. y WHITE, D. R. (2006). Standard Cross-Cultural Sample: online edition. Irvine, University of California. Working Paper series: Social Dynamics and Complexity. Recuperado en: http://escholarship.org/uc/item/ 62c5c02n.

MYERS, H. P. (2001). "Etnomusicología", en Cruces, F., Las culturas musicales: lecturas de etnomusicología, Madrid, Trotta, pp. 19-39.

PELINSKI, R. (2000). Invitación a la etnomusicología: quince fragmentos y un tango. Tres Cantos (Madrid), Akal.

RICE, T. (1994). May it fill your soul: Experiencing Bulgarian music. Chicago, University of Chicago Press.

-, (1997). "Toward a mediation of field methods and field experience in ethnomusicology", en Barz, Gregory F., Cooley, Timothy J. (eds.), Shadows in the Field: New Perspectives for Fieldwork in Ethnomusicology. New York, Oxford University Press, pp. 101-120.

RORTY, R. (2007). Philosophy as Cultural Politics: Philosophical Papers (V. 4). Cambridge, Cambridge University Press.

SÁEZ RUEDA, L. (2002). El conflicto entre continentales y analíticos. Barcelona, Crítica. 
SAMSON, J. (1999). "Analysis in context", en Cook, N. y Everist, M., Rethinking Music. Oxford, New York, Oxford University Press, pp. 35-54.

SIBE. Sociedad de Etnomusicología. Qué es la Sibe. Recuperado en http://www.sibetrans.com/que-es-la-sibe.

SEEGER, A. (2004). Why Suyá Sing: A Musical Anthropology of an Amazonian People. Urbana, Chicago, University of Illinois Press.

SOCIETY FOR ETHNOMUSICOLOGY. What is Ethnomusicology? Recuperado en http://www.ethnomusicology.org/?page=WhatisEthnomusicol.

TITON, J. T. (2009). "Ecology, Phenomenology, and Biocultural Thinking: A Response to Judith Becker". Ethnomusicology 53 (3), pp. 502-509.

—, (2012). "Textual Analysis or Thick Description?", en Clayton, M., Herbert, T. y Middleton, R., The Cultural Study of Music: A Critical Introduction. New York, Routledge, pp. 236-265.

VATIMO, G. (2000). "Prefacio", en D'Agostini, Franca, Analíticos y continentales. Guía de la filosofía de los últimos treinta años. Madrid, Cátedra, pp. 5-15.

WALLIN, N., MERKER, B. y BROWN, S. (eds.) (2000). The origins of music. Cambridge, Massachusetts Institute of Technology Press.

WEBER, M. (1964): Economía y sociedad. Esbozo de sociología comprensiva. México, Fondo de Cultura Económica.

ŽIŽEK, S. (1999): El acoso de las fantasías. México, Siglo XXI. 